\title{
Immunotactoid glomerulonephritis in a child with HIV infection: a
} case report

\author{
Gertruida van Biljon ${ }^{1}$, Melanie Louw ${ }^{2}$ and Leonora Dreyer ${ }^{2}$ \\ 1 Department of Paediatrics, University of Pretoria, Faculty of Health Sciences, P.O. Box 667, Pretoria, 0001, South Africa \\ 2 Department of Anatomical Pathology, National Health Laboratory Services, Tshwane Academic Division, University of Pretoria, \\ P.O. Box 667, Pretoria, 0001, South Africa
}

\section{Abstract}

Renal disease is a common complication of human immunodeficiency virus (HIV) infection in adults. In children, however, HIV pathology of the gastrointestinal and respiratory tracts predominates, whereas renal disease is often an incidental finding. In this report, we describe a child with stage III HIV infection associated with immunotactoid glomerulonephritis (IT). The patient was referred for investigation of idiopathic nephrotic syndrome. Electron microscopy of his renal biopsy specimen revealed numerous electron-dense microtubular deposits, arranged in parallel fashion, consistent with a diagnosis of IT. He received highly active antiretroviral therapy (HAART) as well as corticosteroids and is currently in remission. To our knowledge, this is the first report of IT in a child.

\section{Introduction}

Renal disease is a common complication of human immunodeficiency virus (HIV) infection in adult patients [1]. In children with HIV infection, on the other hand, pathology of the gastrointestinal and respiratory tracts predominates, whereas renal disease is often an incidental finding. Occasional cases of fibrillary or immunotactoid glomerulonephritis (IT) have been described in the literature, mostly in adults with HIV, accompanied by hepatitis C infection [2, 3]. In this report, we describe a child with stage III HIV infection with associated IT. To our knowledge, this is the first report of this condition in a child.

\section{Case report}

A 6-year-old black male child was referred for investigation of idiopathic nephrotic syndrome. $\mathrm{He}$ had a history of recurrent febrile chest infections. The infections were nonremitting, and he was coughing constantly. He also had intermittent episodes of self-limiting acute gastroenteritis. There was no history of dysuria, frequency, macroscopic hematuria, or previous throat or skin infections. The mother denied any administration of home remedies, drugs, or traditional-healer medications. There was no family history of kidney disease. Clinical examination revealed an acutely ill, febrile child with generalized edema and lymphadenopathy. He had anemia, parotid enlargement, hepatosplenomegaly, and ascites. There were diffuse chest crepitations but no cyanosis. His blood pressure was 102/68 $\mathrm{mmHg}$ (normal).

Special investigations Our investigations revealed the following: Urine dipstick test: $3+$ proteinuria, 1+ hematuria, 2+ leucocytes, and 2+ nitrites; urine microscopy: 75 pus cells and 20 red blood cells per high-power field; urine culture: Escherichia coli $>10^{5}$ colonies $/ \mathrm{ml}$, sensitive to amoxicillinclavulanic acid; urine protein-creatinine ratio: varied from 2.0 to $3.8 \mathrm{~g} / \mathrm{mmol}$ (normal: $<0.02 \mathrm{~g} / \mathrm{mmol}$ ); serum creatinine: $36 \mu \mathrm{mol} / \mathrm{l}$ (normal: 53-80 $\mu \mathrm{mol} / \mathrm{l}$ ); serum albumin level: $8 \mathrm{~g} / \mathrm{l}$ 
(normal 32-47); serum cholesterol: $8.1 \mathrm{mmol} / \mathrm{l}$ (normal <50); full blood count: normocytic anemia $(\mathrm{Hb}: 9.7 \mathrm{~g} / \mathrm{dl})$, normal 10.7-15.1; absolute CD4 count 1,180.106/l (normal 500-2010); serum complement levels: decreased C3, $0.71 \mathrm{~g} / \mathrm{l}$ (normal: 0.9-1.8) and C4, $0.14 \mathrm{~g} / \mathrm{l}$ (normal: 0.2-1.0); serology for hepatitis $\mathrm{B}$ and $\mathrm{C}$, cytomegalovirus (CMV) and syphilis: negative. Tests for autoantibodies and antinuclear factor were also negative. The HIV enzyme-linked immunosorbent assay (ELISA) was positive, confirmed by a viral load of $91,000 \mathrm{copies} / \mathrm{ml}$.

Kidney sonar revealed bilateral enlarged kidneys. Chest X-ray examination revealed hilar lymphadenopathy and diffuse nodular interstitial infiltrates. A renal biopsy was performed at this stage. Histology revealed enlarged glomeruli with foci of mesangial cell proliferation and segmental thickening of the capillary membranes (Fig. 1). Congo red stain was done to exclude amyloid deposits (Fig. 2), and silver stains confirmed the presence of thickened membranes with occasional areas of "tram tracking". Immunohistochemical stains showed focal deposition of IgG and IgM. With electron microscopy, subepithelial deposits were found (Fig. 3a), and on higher magnification, hollow microtubular deposits arranged in parallel fashion were seen (Fig. 3b,c). The diameter ranged from 14.8 to $22.3 \mathrm{~nm}$. Following the biopsy, several further investigations were performed: cryoglobulins were negative; serum protein electrophoresis revealed a few restriction bands on a polyclonal background in the gamma region; bone marrow aspirate showed a reactive bone marrow with dyserythropoiesis and an increase in reticulum cells, as well as macrophage activity with signs of hemophagocytosis; plasma cells were prominent but not numerically increased.

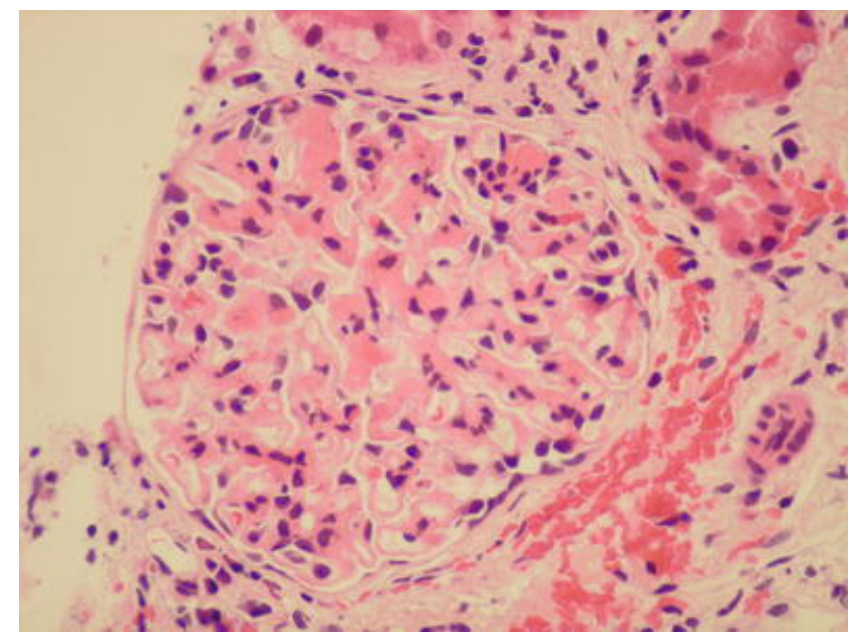

Fig. 1 Hematoxylin and eosin (H\&E) stain of the glomeruli ( $\times 40$ magnification) demonstrating thickened basement membranes 


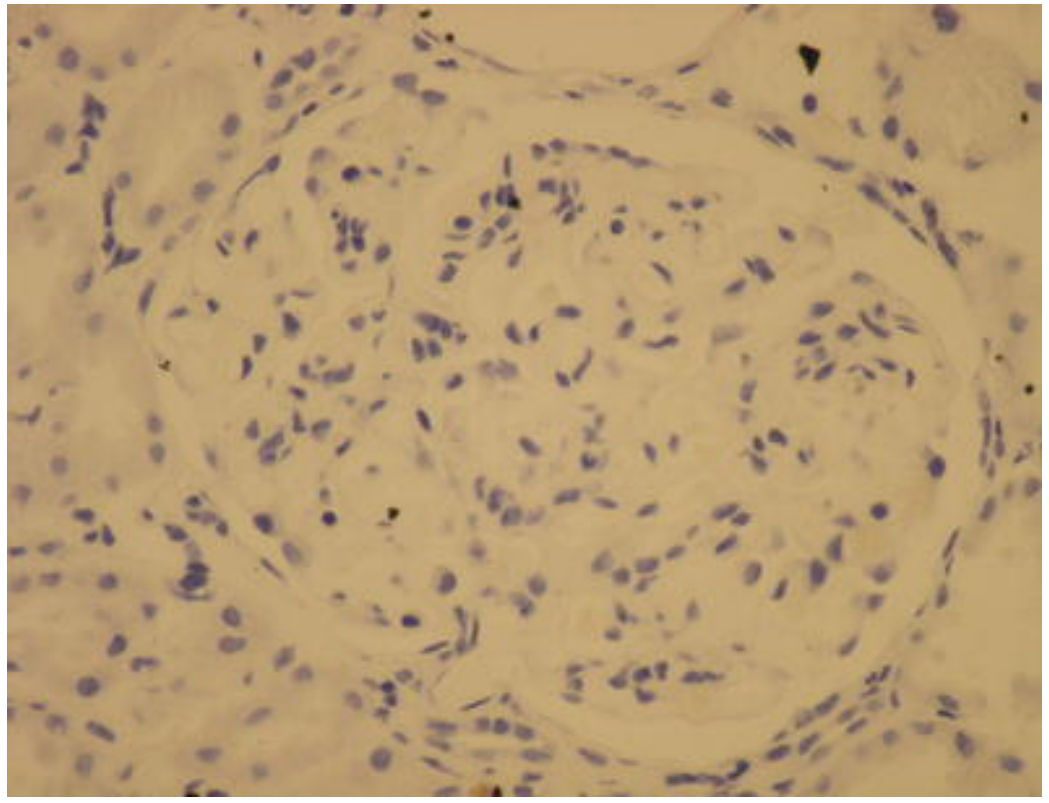

Fig. 2 Congo red stain confirming the absence of amyloid in the glomeruli
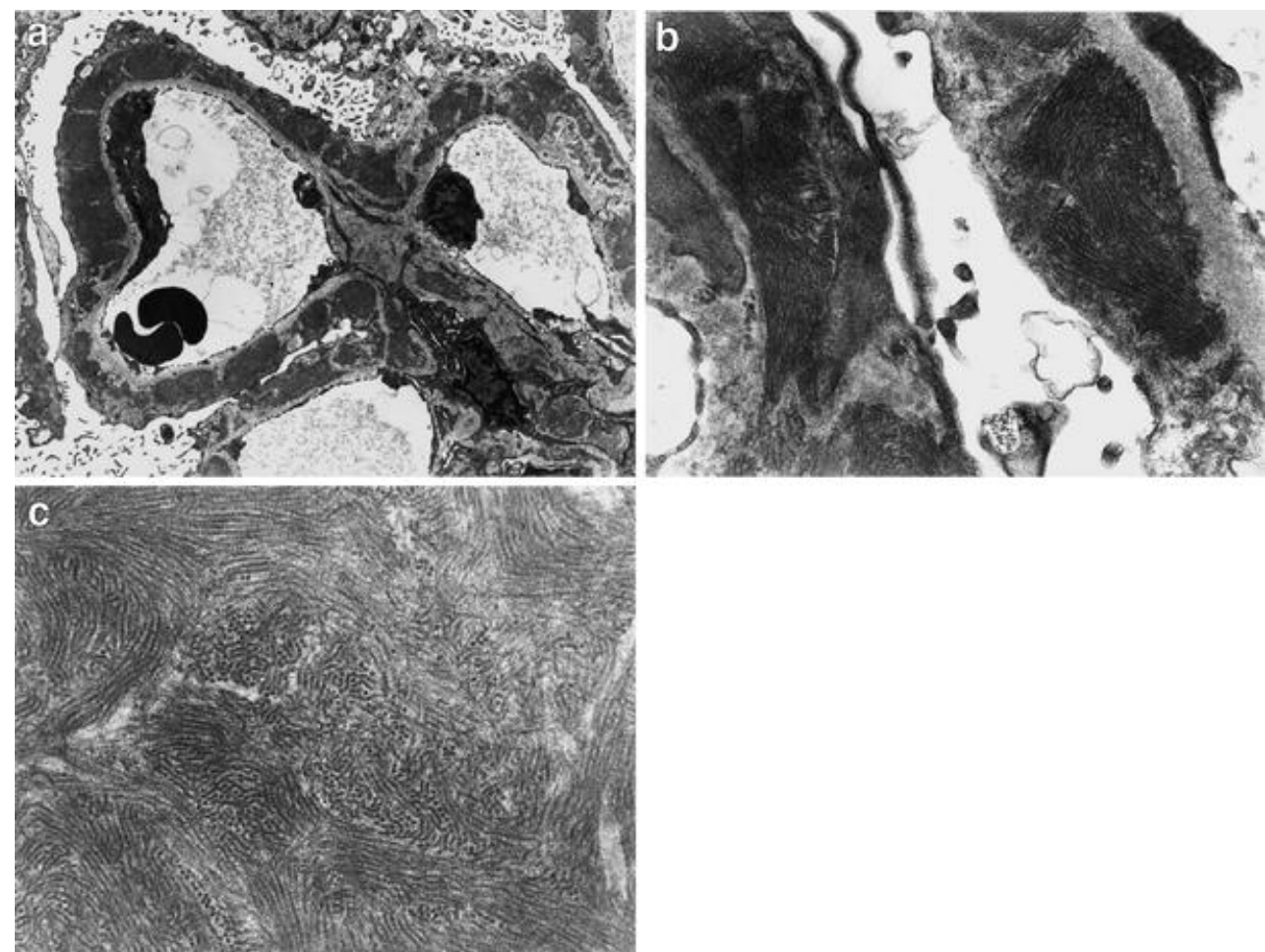

Fig. 3 a Low-magnification electron microscopy demonstrating the extensive electron-dense deposits in the subepithelial side of the membrane $(\times 3,300)$. b Deposits consisting of microtubuli arranged in a parallel fashion $(\times 18,300)$. c Hollow microtubules with lumen diameters ranging from $14.8 \mathrm{~nm}$ to $22.3 \mathrm{~nm}(\times 26,900)$. 


\section{Discussion}

In 1977, Rosenmann and Eliakim [4] reported a glomerulopathy characterized by fibrillary deposits in the glomeruli resembling amyloid in appearance and location but differing by virtue of their larger size and lack of reactivity with conventional reagents used to identify amyloid. More recently, two distinct glomerulopathies with electron-microscopic nonamyloid fibrillary deposits were described, namely, fibrillary glomerulonephritis (FGN) and IT [5]. The distinction between FGN and IT is based on morphological differences visible on electron microscopy [5-7]. In IT, parallel-arranged hollow microtubular glomerular deposits with diameters ranging from 16.8 to $90.0 \mathrm{~nm}$ are found. In FGN, electron microscopy reveals randomly arranged fibrillary deposits with much smaller diameters ranging from 10.4 to $24 \mathrm{~nm}$ in the glomeruli. The deposits stain positively for IgG and C3. FGN is a rare condition that occurs in less than $1 \%$ of renal biopsy specimens [8]. Clinically, patients usually present with nephrotic-range proteinuria, microscopic hematuria, and renal insufficiency.

As more patients with renal lesions containing nonamyloid fibrillary deposits are being identified, controversy has arisen about the histological classification $[3,5,6]$. Proponents of the view that FGN and IT are related entities have argued that the morphological differences are merely a function of fibril diameter and the resolution of the electron microscope rather than intrinsic physicochemical properties or etiological differences. The alternative proposal, based on morphological and clinical features, is that FGN and IT are two separate entities. Advocates of the latter proposal also believe that the morphological classification is of clinical significance. Patients with IT are older than those with FGN and more commonly suffer from systemic diseases such as lupus erythematosus, cryoglobulinemia, chronic lymphocytic leukemia and other gammopathies [5] It may, however, present as de novo IT. In the literature, chronic renal impairment was reported more consistently in association with IT, whereas end-stage renal disease (ESRD) occurred more often in association with FGN [5].

HIVIAIDS is characterized by a marked abnormality in cellular immunity associated with recurrent opportunistic infections and Kaposi sarcoma. Many different organ systems may be involved in $\mathrm{HIV}$, including the kidney, the involvement of which may result in acute or chronic renal disease. The most common HIV-associated histopathological renal lesions in adults are focal segmental glomerulosclerosis and HIV-associated immune-complex glomerulonephritis [9]. The histopathological findings in renal biopsy specimens of HIV-positive patients strongly suggest a persisting immune-mediated etiology, most likely due to accompanying chronic infections or HIV infection per sè [9]. Excessive quantities of circulating immune complexes lead to its deposition in various organs, especially in the renal glomeruli. Supportive of an immune-mediated pathogenesis is the finding of $\lg \mathrm{A}$, IgM, and $\lg$ antibodies complexed to HIV p24 antigen eluted from renal biopsy tissue [9]. The role of cellular incorporation of HIV genomic products in the pathogenesis of renal disease is unknown. Two potential pathophysiologic mechanisms of HIV-associated immunecomplex glomerulonephritis are proposed: (1) deposition of immune complexes acting as the inciting factor, or (2) in situ immune-complex formation initiated by the presence of HIV genome in renal tissue.

In our pediatric HIV group, disease of the gastrointestinal and respiratory tracts is much more common than is renal pathology, which is most often an incidental finding. IT appears to be rare in the HIV pediatric population. A possible explanation is the high incidence of other associated serious infections, leading to an early demise in untreated patients, before the development of renal disease. HIV-associated glomerular lesions causing nephrotic syndrome have therefore not been adequately studied. 
In the current patient, the most probable source of immune modulation was the HIV infection itself. Nine months after initiation of combined highly active antiretroviral therapy (HAART) (d4T, 3TC and Kaletra) and corticosteroid treatment, remission of proteinuria was achieved. The most recent serum albumin level was $37 \mathrm{~g} / \mathrm{l}$, serum creatinine $29 \mu \mathrm{mol} / \mathrm{l}$, and urine protein:creatinine ratio $0.01 \mathrm{~g} / \mathrm{mmol}$ (normal). HIV viral load was undetectable, and CD4 count was $1.441 \times 10^{6} / 1$. It seems that early treatment with HAART and corticosteroids for HIV-associated IT may result in complete cure of the nephrotic syndrome provided therapy is commenced prior to the development of glomerulosclerosis.

\section{References}

1. Monahan M, Tanji N, Klotman PE (2000) HIV-associated nephropathy: an urban epidemic. Semin Nephrol 21(4):394-402

2. Haas M, Rajaraman S, Ahuja T, Kittaka M, Cavallo T (2000) Fibrillary/immunotactoid glomerulonephritis in HIV positive patients: a report of three cases. Nephrol Dial Transplant 15(10):1679-1683

3. Rosenstock JL, Markowitz GS, Valeri AM, Sacchi G, Appel GB, D’Agati VD (2003) Fibrillary and immunotactoid glomerulonephritis: Distinct entities with different clinical and pathologic features. Kidney Int 63(4):1450-1461

4. Rosenmann E, Eliakim M (1977) Nephrotic syndrome associated with amyloid-like glomerular deposits. Nephron 18(5):301-308

5. Fogo A, Qureshi N, Horn RG (1993) Morphological and clinical features of fibrillary glomerulonephritis versus immunotactoid glomerulopathy. Am J Kidney Dis 22(3):367-377

6. Alpers CE (1993) Fibrillary glomerulonephritis and immunotactoid glomerulopathy: Two entities, not one. Am J Kidney Dis 22(3):448-451

7. Alpers CE (1992) Immunotactoid (microtubular) glomerulopathy: An entity distinct from fibrillary glomerulonephrits. Am J Kidney Dis 19(2):185-191

8. Iskandar SS, Falk RJ, Jennette JC (1992) Clinical and pathologic features of fibrillary glomerulonephritis. Kidney Int 42(6):1401-1407

9. Weiner NJ, Goodman JW, Kimmel PL (2003) The HIV-associated renal diseases: Current insight into pathogenesis and treatment. Kidney Int 63(5):1618-1631 